\title{
Educação permanente: componente estratégico para a implementação da política nacional de atenção oncológica
}

\author{
Simone Paes Vincent*
}

\section{Resumo}

A reflexão acerca da formação de Recursos Humanos para o trabalho na atenção oncológica se faz, neste texto, a partir do conceito de Educação Permanente em Saúde conforme enunciado na década de 1980 pela Organização Panamericana de Saúde (OPAS). O artigo propóe a estruturação de uma Rede de Educação Permanente como componente estratégico da Política Nacional de Atenção Oncológica, constituída pela ação sinérgica dos diversos atores envolvidos, na perspectiva da articulação de educação e trabalho para o enfrentamento do câncer como problema de saúde pública, no contexto do Sistema Único de Saúde e pautada pelos parâmetros da universalidade, eqüidade e integralidade, ordenadores do Sistema.

Palavras-chave: Educação Permanente em Saúde; Educação e trabalho; Gestão de recursos humanos; Qualidade da atenção à saúde. 


\section{INTRODUÇÃO}

O Pacto pela Saúde 2006 - Consolidação do SUS ${ }^{1}$, em suas Diretrizes Operacionais ${ }^{2}$, estabelece os princípios da Educação Permanente como "orientadores da formação e do desenvolvimento de trabalhadores para o setor". A Educação Permanente, na valorização do aspecto educacional inerente às atividades, conforme Schwartz ${ }^{3}$ chamadas de industriosas, expressa o reconhecimento da vinculação inextricável de gestão e educação como eixo central para a elaboração de estratégias político-gerenciais e educacionais com vistas à qualidade na atenção à saúde. Por essa lógica, a articulação de educação e trabalho deve orientar a formação e a gestão, comprometidas não apenas com a qualidade técnica, mas conjugadas às necessidades da população em direção ao aperfeiçoamento do Sistema de Saúde do país.

É na perspectiva da Educação Permanente em Saúde que, neste artigo, busca-se refletir acerca da formação de pessoas para o enfrentamento do câncer como uma questão de saúde pública, objeto de Política de Estado, em direção a uma Atenção Oncológica integral, pautada pelos princípios ordenadores do SUS.

\section{EdUCAÇÃo PERMANENTE: ESTRATÉGIA PARA A TRANSFORMAÇÃO DAS PRÁTICAS EM SAÚDE}

O conceito de Educação Permanente surge na década de 1980, a partir da percepção do reduzido impacto dos programas de capacitação profissional e de atualização de conhecimentos (Educação Continuada - EC) na resolução dos problemas da prática do trabalho e na qualidade dos serviços prestados na saúde.

A lógica da EC, centrada na atualização de conhecimentos de caráter individual, passa a ser questionada em um novo conceito de educação com caráter relacional - a Educação Permanente (EP), que pressupõe educação no trabalho, pelo trabalho e para o trabalho e considera que a gestão do conhecimento e a gestão do trabalho são processos indissociáveis ${ }^{4}$.

Em uma visão de "educação continuada", o enfrentamento dos problemas na prática profissional se faz pela atualização, por categorias profissionais, de conhecimentos/capacitação técnica a partir de um "diagnóstico" das deficiências na prestação de serviços, habitualmente feito pelas gerências de forma não integrada, sem dar voz àqueles que se encontram na ponta assistencial do serviço e são, em última análise, os responsáveis pela sua operacionalização.

Por essa lógica, se os esforços não resultam efetivos na resolução dos supostos problemas do serviço, há apenas dois caminhos a seguir, nos programas de capacitação/atualização:

- o redirecionamento do programa, caso se assuma que o treinamento se desenvolveu conforme o esperado, com um novo diagnóstico gerando novos cursos de atualização;

- a intensificação da capacitação, caso se considere insuficiente o treinamento executado.

Em qualquer destes cenários, entretanto, a relação custo/benefício é muito alta, resultando na exaustão de recursos humanos e financeiros, com tendência a entrar em um círculo vicioso: treinamento - redirecionamento/ intensificação - treinamento, no qual o objetivo final, evoluir concretamente para a melhor prestação de serviços, se perde, quase sempre, irremediavelmente.

Adicionalmente, a pouca articulação das diversas gerências responsáveis por um mesmo programa, em sua compartimentalização por categorias profissionais, associa-se ao fato de os profissionais a serem atualizados nunca, ou quase nunca, participarem do seu planejamento.

Dessa combinação resultam, muitas vezes, programas fragilizados em sua estrutura, aplicados por profissionais não integrados, pouco conscientes do objetivo geral do projeto, afinados, quando muito, com os aspectos técnicos pontuais do conteúdo a ministrar.

Os profissionais em treinamento, por sua vez, reduzidos a meros espectadores, muitas vezes convocados às pressas para participar dos cursos de atualização, apresentam-se descomprometidos com o projeto de capacitação, ou mesmo em franca (ou velada) oposição.

O descompasso e a fragmentação no planejamento e na operacionalização são graves limitadores nos programas de capacitação/atualização. Os participantes (em todos os níveis, mas especialmente aqueles que assistem aos cursos) são privados da visão geral do projeto, grande obstáculo na construção de significado que daria sentido e integraria, afinal, os cursos ao contexto do programa, e este, ao objetivo último de um projeto de educação institucional: a melhor qualidade do serviço prestado.

Assim, gestores e coordenadores desenvolvem programas de capacitação/atualização nos quais planejamento e execução são centrados nos procedimentos técnicos que os profissionais deverão executar, procedimentos esses identificados com a excelência da atuação profissional que, por sua vez, asseguraria a qualidade pretendida. A ênfase nos aspectos 
quantitativos é evidente, com pouca ou nenhuma referência aos aspectos qualitativos dos projetos. Os planejamentos quantificam detalhadamente cargas horárias, disciplinas, temas a atualizar e a avaliação dos programas é expressa em relatórios ricos em dados numéricos: número de alunos inscritos, de cursos realizados, de procedimentos treinados, taxa de comparecimento, absenteísmo, vagas ociosas, setores contemplados pelo programa. Pouco ou quase nunca se faz referência ao impacto do treinamento sobre a qualidade da prática clínica ou sobre as mudanças alcançadas nas condições de saúde da população sob os cuidados dos profissionais treinados.

Dificilmente, programas educacionais constituídos dessa forma poderão corresponder às demandas e problemas da prática profissional e, ainda menos, à formação segundo as necessidades do Sistema Único de Saúde (SUS), conforme o estabelecido para o setor saúde: no Brasil, o Ministério da Saúde tem a responsabilidade, por definição constitucional e da Lei Orgânica da Saúde , de estimular e ordenar o processo da formação de trabalhadores em saúde.

As estratégias de educação permanente (EP) no setor público vêm sendo elaboradas na América Latina, conforme assinalado, desde a década de 1980, impulsionadas na saúde pela Organização Pan-americana de Saúde (OPAS) como alternativa político-pedagógicagerencial que efetivamente dê conta da melhoria na prestação de serviços na sua integralidade em contraponto às intervenções não coordenadas e pouco eficazes resultantes dos projetos de educação continuada ${ }^{4}$. Nos projetos pautados pelo conceito de educação permanente, os problemas da prática, as demandas para uma prestação de serviços de qualidade são objeto de reflexão coletiva pelos sujeitos da atenção à saúde, a partir da qual são definidas as necessidades de intervençôes político-educativas e outras, referenciadas à esfera da gestão.

O "problema", categoria central na EP, é construído e não "diagnosticado": é a partir dos olhares de todos os atores envolvidos na prestação de serviço que o problema se desenha. Esse delineamento se processa continuamente, renovando a compreensão de necessidades de saúde, de práticas profissionais e de organização do trabalho que evoluem, em transformação mútua e permanente, em direção a uma noção de qualidade na atenção à saúde pautada pela compreensão desse processo de mudança contínua.

A configuração do problema, em conformidade com a complexidade do trabalho em saúde, encontra-se no centro do projeto educacional da organização, alimentando o processo de construção de conhecimento, não apenas técnico, mas também relativo ao processo de trabalho e à sua gestão. Parte-se assim do pressuposto de que nem todos os problemas resultam da falta de conhecimentos técnicos dos profissionais de saúde.

O conhecimento dessa forma construído ganha significado para aqueles que atuam nos serviços de saúde. A construção do conhecimento acontece no processo de trabalho, a ele alimenta e é a partir dele reconstruído ${ }^{4,6}$

Um novo círculo se inicia, então: construção de conhecimento no e por meio do processo de trabalho modificação nas práticas e necessidades da atuação profissional - reconstrução do conhecimento. Por meio desse processo, de caráter permanente, são instituídas estratégias/intervenções educacionais e de gestão.

Essa concepção de educação permanente carrega, como processo que alia educação e gestão, um enorme potencial transformador na prática do trabalho e na qualidade do serviço prestado.

No Brasil, o Ministério da Saúde constituiu, em 2003, a Secretaria de Gestão do Trabalho e da Educação na Saúde (SGTES) com o objetivo de inserir os conceitos de gestão do trabalho e da educação na saúde a partir dos princípios e diretrizes do SUS, e promover a qualidade e a humanização nos serviços prestados pelo Sistema Único de Saúde, assumindo o papel, estabelecido pela legislação, de gestor federal do SUS na formulação de políticas orientadoras da gestão, da formação, do desenvolvimento, da educação permanente e da regulação do trabalho em saúde no Brasil.

A SGETS/MS, ao propor a agregação entre desenvolvimento individual e institucional, serviços, gestão setorial e controle social, constitui-se na perspectiva da transformação da organização do trabalho, dos processos formativos, das práticas de saúde e das práticas pedagógicas em relações orgânicas e permanentes do sistema de saúde, em suas várias esferas de gestão, com as instituiçōes formadoras ${ }^{7}$.

\section{A ESTRATÉGIA DE EDUCAÇÃO PERMANENTE E O CONTROLE DO CÂNCER NO BRASIL}

A publicação recente da Política Nacional de Atenção Oncológica $(\mathrm{PNAO})^{8}$ vem trazer visibilidade ao câncer como problema de saúde pública: segunda causa de morte no país ${ }^{9}$, tem taxas de mortalidade crescentes apesar dos esforços intensivos e dos grandes investimentos em formação de recursos humanos, em equipamento e em desenvolvimento tecnológico para a 
prevenção, controle e assistência nessa área.

A Política Nacional de Atenção Oncológica, estruturando-se em redes estaduais e regionais de atenção oncológica, na lógica da linha de cuidados progressivos em saúde, busca romper com a organização "em pirâmide" das iniciativas para o enfrentamento do problema do câncer. Entre seus eixos norteadores, inserem-se a qualificação da assistência e a promoção da educação permanente dos profissionais de saúde envolvidos com a implantação e implementação da política, em consonância com os princípios da integralidade e da humanização. Nessa perspectiva, a PNAO tem papel estratégico para a consolidação da integralidade da atenção de todo o SUS, com o potencial de promover a interlocução das diversas esferas gestoras e assistenciais, em todos os seus níveis, desde a atenção básica até os cuidados pós-hospitalares de reabilitação/paliação.

O cenário da atenção oncológica apresenta, entretanto, desafios enormes para a transformação das práticas na atenção à saúde na ótica do enfrentamento do problema do câncer de forma integral, em direção aos princípios norteadores do SUS. O desenvolvimento de estratégias para o controle do câncer depende da abordagem a problemas que afetam desde os mecanismos de formulação das políticas de saúde até a mobilização social, a organização e o desenvolvimento das ações e serviços de saúde e a geração e difusão de conhecimento. Na perspectiva da superação de tais problemas, o Instituto Nacional de Câncer (INCA), órgão do Ministério da Saúde responsável pela formulação da Política de Controle do Câncer no Brasil, estabelece, desde 2004, uma política indutiva de estruturação, em nível nacional, da Rede de Atenção Oncológica (RAO), na compreensão de que, para o combate ao câncer, é indispensável que se promova a integração dos diferentes atores da atenção oncológica na formulação de políticas e desenvolvimento de ações com vistas à mobilização social, à produção de conhecimento e à assistência à saúde em todo o espectro da linha de cuidados nessa área.

Cumprir com um compromisso de tal natureza exige a adesão à estratégia de Educação Permanente em Saúde de forma plena, incorporada à própria estrutura da Rede de Atenção Oncológica e à formação de recursos humanos para a rede. A Educação Permanente, compreendida como processo de vinculação de educação e trabalho, é ferramenta estratégica para a reconfiguração das práticas de formação, gestão e assistência, para modificar a lógica da formulação de políticas públicas e fortalecer o controle social.
Nesse sentido constitui-se a proposta de criação de uma Rede de Educação Permanente em Atenção Oncológica (REPAO) que se insere na RAO com a potência para reforçar e ampliar a vinculação entre trabalho, gestão e educação, no contexto do Sistema Único de Saúde e na perspectiva dos parâmetros da universalidade, eqüidade e integralidade, ordenadores do sistema. Por essa ótica, os hospitais de ensino e universitários são atores estratégicos na construção da REPAO: atuam nessa rede em cooperação efetiva como instituições voltadas à produção e difusão do conhecimento, cuja ação coletiva e sinérgica é capaz de potencializar os esforços para a promoção da pesquisa, da inovação tecnológica e da formação de recursos humanos comprometidas com o controle do câncer no país.

A formação para a atenção oncológica deve promover o desenvolvimento de competência profissional adequada às práticas de trabalho nessa área da saúde em toda a sua complexidade e abrangência, sempre com base nas melhores evidências científicas existentes, apoiadas por um sólido julgamento clínicoepidemiológico e princípios éticos, e centrada na melhoria do cuidado em câncer no país. $\mathrm{O}$ conhecimento e a prática técnica têm lugar, mas como parte de um aprendizado significativo, inseridos no processo de trabalho, contextualizados por profissionais conscientes da natureza social de sua atividade profissional ${ }^{10}$.

Nesse cenário, a Rede de Educação Permanente em Atenção Oncológica tem o compromisso de articular gestão e trabalho sob a ótica da integralidade da atenção de forma inclusiva de todos os atores dos diversos segmentos da sociedade envolvidos na questáo do câncer. Em sua inserção na $\mathrm{PNAO}$, é estratégica para a promoção do diálogo nas diferentes esferas do SUS indispensável para que de fato seja possível "desenvolver estratégias coerentes com a política nacional de promoção da saúde voltadas para a identificação dos determinantes e condicionantes das principais neoplasias malignas e orientadas para o desenvolvimento de ações intersetoriais de responsabilidade pública e da sociedade civil que promovam a qualidade de vida e saúde, capazes de prevenir fatores de risco, reduzir danos, proteger a vida, de forma a garantir a eqüidade e a autonomia de indivíduos e coletividades"8.

Os princípios norteadores da REPAO consolidarse-ão, concretamente, por meio de projetos que se desenvolvam em processos de Educação Permanente, de educação articulada ao planejamento institucional e ao controle social, da transformação da realidade e seus determinantes, fundamentada na educação, no processamento de situações-problema extraídas do 
espaço de trabalho e do campo social ${ }^{11}$.

\section{ESTRATÉGIAS POLÍTICO-PEDAGÓGICO-GERENCIAIS PARA 0 DESENVOLVIMENTO DA REPAO}

\section{Trabalho e educação em saúde: implicações na Atenção Oncológica}

O mundo contemporâneo, da globalização, da vida on-line, da informação em tempo real, imprime, com suas demandas e contradiçôes, modificaçõos profundas em todos os setores da sociedade atual. O mundo do trabalho, no qual se concentra a maior parte das atividades humanas, é a esfera na qual essas mudanças são percebidas mais ostensiva e dramaticamente.

As transformações nas relações de trabalho estão ocorrendo no dia-a-dia das organizações em todos os setores profissionais. A natureza dessas novas relaçōes rompe com a lógica da teoria clássica de administração ${ }^{12}$, alicerce do modelo industrial de gestão e formação profissional, norteadora da "gestão científica" de recursos humanos desde o final do século XIX, predominante ainda hoje.

$\mathrm{Na}$ América Latina, as Escolas de Saúde Pública, formadoras dos gestores em saúde, foram fortemente influenciadas pela concepção, característica da "ciência administrativa" clássica, de profissionalização da administração do trabalho, com ênfase nas gerências ${ }^{12}$. Em um modelo hospitalocêntrico de atenção à saúde, os serviços de saúde latino-americanos incorporaram o pensamento administrativo desenhado para a produção de bens por organizaçôes com fins lucrativos em países desenvolvidos e gerenciando suas instituições como se fossem "fábricas para a produção de consultas e egressos"6.

Entretanto, no mundo do trabalho contemporâneo, a atividade profissional exige, para se desenvolver, parâmetros de adequação às mudanças contínuas, características de um novo modelo teórico "pósindustrial"13. O profissional moderno se define pela competência profissional em seu sentido mais abrangente; tem pleno domínio da técnica, mas é igualmente versátil, articulado, capaz de mobilizar conhecimentos, refletir e tomar decisôes ${ }^{14}$.

No Brasil, essa concepção já se incorpora ao discurso do Estado, especialmente em suas políticas relativas à educação e à formação de recursos humanos. Assim, o Ministério da Educação (MEC) extinguiu, em 1996, o currículo mínimo em todos os níveis da educação e estabelece parâmetros e diretrizes curriculares norteados pelo desenvolvimento de competências. Instituiu a seguir, em 1997, a Política Nacional de Educação
Profissional que estabeleceu a Educação Profissional, inicialmente com três níveis: básico, independente de escolaridade pré-estabelecida; técnico, condicionado à conclusão da educação de nível médio; tecnológico, formação de nível superior.

O Ministério da Saúde, por sua vez, implementou programas tendo em vista a formação de profissionais adequados às demandas do mercado de trabalho ${ }^{15}$, como o Programa de Incentivo às Mudanças Curriculares nos Cursos de Medicina (PROMED) e o Projeto de Profissionalização dos Trabalhadores da Área de Enfermagem (PROFAE) e evoluiu na direção de articular formação e trabalho com a criação, em 2003, da Secretaria de Gestão do Trabalho e da Educação na Saúde, instância propositora da Política de Educação Permanente em Saúde para o SUS7.

É preciso, no entanto, ter consciência da influência ainda predominante do pensamento administrativo clássico nas práticas administrativas em Saúde Pública e do conseqüente impasse vivido no momento atual: o hiato entre formação, gestão e prática profissional, evidente mesmo nas grandes organizaçôes privadas, mais sensíveis a propostas inovadoras de formação e gestão, e muito mais profundo nas organizações públicas de saúde ${ }^{6}$, compreensivelmente mais lentas na implementação das transformações estruturais que se impõem no mundo do trabalho contemporâneo. $\mathrm{O}$ enfrentamento desse impasse é decisivo para a implementação de estratégias político-pedagógicogerenciais que verdadeiramente sustentem o processo de trabalho em saúde pública na sua integralidade.

$\mathrm{Na}$ formação profissional para a atenção oncológica, as estratégias pedagógicas orientaram-se, historicamente, à capacitação de profissionais da saúde para a assistência, como na saúde em geral, no modelo clássico de gestão e formação "científicas": treinamento estritamente técnico, desvinculado dos aspectos subjetivos da prática profissional, do contexto no qual se dá essa prática e dos princípios norteadores da atenção à saúde para o SUS.

Nesse momento de implementação da Política Nacional de Atenção Oncológica, com a proposição de uma Rede de Atenção Oncológica, não se pode desprezar a tendência de que velhos e tradicionais modelos se imponham na construção de "propostas pedagógicas de capacitação", instituindo programas para a difusão de tecnologia, com capacitação e formação de multiplicadores, como tantas vezes se viu acontecer em toda a América Latina para, logo em seguida, questionarse a eficácia dessas medidas - freqüentemente, na pressuposição de que a qualificação técnica foi 
insuficiente, gerando novos projetos de capacitação, num círculo de insucessos sem fim ${ }^{6}$.

$\mathrm{Na}$ compreensão de que: (i) toda estratégia pedagógica visando um exercício profissional deve ter sólida conexão com a prática do trabalho; (ii) essa vinculação deve constituir-se em elemento central das políticas públicas de gestão do processo de trabalho em saúde; considerase estratégico, na consolidação da Rede de Atenção Oncológica no país, que instituições formadoras, movimentos sociais, gestores, serviços de saúde envolvidos na atenção oncológica e hospitais de ensino e universitários certificados pelo MEC/MS integrem, em ação sinérgica, a Rede de Educação Permanente em Atenção Oncológica (REPAO), instância a constituir-se com vistas à indução de transformações nas esferas da gestão, práticas profissionais e formação na atenção oncológica sob a ótica da qualidade do cuidado.

\section{0 processo de Educação Permanente - ações estratégicas para a REPAO}

Em conformidade com o proposto, uma Rede de Educação Permanente deve ser compreendida para além de sua dimensão de preparação para o trabalho - situação em que estaria sob responsabilidade apenas dos profissionais da educação - mas como resultante da pactuação entre os atores-chave que a conformam de maneira a construir uma sólida base de atuação sinérgica intra e interinstitucional. Gestores da atenção oncológica, controle social, serviços e instâncias comprometidas com a formação nesse campo são, nesse sentido, autores do projeto político-pedagógico-gerencial.

Sob essa concepção, a REPAO se constitui como um sistema descentralizado de construção de demandas educativas e gerenciais, de processamento ascendente e descendente, que responda aos graus de autonomia alcançados pelos municípios, em seus processos de formação e de EP, e que assegure o apoio necessário do Estado ao desenvolvimento da Rede de Atenção Oncológica. Nessa Rede, os atores envolvidos operam em "colaboração"16, processo articulado de trabalho, para:

- promover e apoiar a interlocução qualificada de gestores, agentes executores, formadores e usuários na pactuação e no desenvolvimento de planos de trabalho loco-regionais para a Rede de Atenção Oncológica;

- mediar a interlocução de gestores e instituições formadoras na identificação e na resposta às demandas educativas e gerenciais na referida Rede;

- estabelecer indicadores e propor estratégias para a adequação constante das políticas de desenvolvimento de RH e de gestão do trabalho na perspectiva da qualidade da atenção oncológica na integralidade do SUS; - promover a formação de formadores em práticas pedagógicas orientadas ao aprendizado significativo e ao desenvolvimento de competência profissional; - estabelecer política indutiva para estimular a produção de conhecimento de forma a subsidiar a formulação de políticas, a gestão da assistência e da educação no âmbito da atenção oncológica, tanto em seu aspecto de intervenção como naquele que reflete o desempenho do SUS no atendimento às necessidades nesta área; - pactuar critérios mínimos para a acreditação de instituições parceiras na Rede de Atenção Oncológica; - formar docentes, gestores e profissionais na perspectiva da educação permanente para a atenção oncológica descentralizada, ascendente, multidisciplinar, com visão abrangente da prática profissional, da qualidade técnica indissociável da ética e do compromisso social.

O estabelecimento do processo de Educação Permanente exige a qualificação dos atores envolvidos para a construção e o processamento de problemas que se configurem verdadeiramente relevantes para a Atenção Oncológica integral. Nesse sentido, é fundamental que se formem facilitadores de EP:

- entre os gestores, permitindo a identificação dos problemas que enfrentam na atenção oncológica e construindo as demandas das esferas de gestão em níveis central e municipal a serem processadas em conjunto com os demais atores;

- nas instituições formadoras que hoje apresentam potencial para atender às demandas educativas no campo do controle do câncer, com ênfase nas dimensões metodológicas do processo e suas implicaçóes para as práticas de formação e capacitação;

- nas equipes de trabalho em todos os níveis da atenção oncológica, em seus diferentes cenários.

\section{DESAFIO DA OPERACIONALIZAÇÃO}

A implementação de uma Rede de Educação Permanente com vistas à indução de transformações nas esferas da gestão, das práticas profissionais e da formação, na perspectiva da melhoria do controle e da prevenção do câncer no Brasil representa um enorme desafio. Na concepção de que enfrentá-lo é condição fundamental para que efetivamente se estabeleça a Política Nacional de Atenção Oncológica, considera-se como aspecto central o comprometimento dos diversos atores na articulação e na ampliação de parcerias em ação conjunta para a melhoria do cuidado nessa área. Nesse cenário, reconhece-se que a integração das instâncias da atenção à saúde, já condicionada a interesses 
políticos e econômicos diversos, depende, para a sua operacionalização, de alternativas metodológicas apenas anunciadas, ainda por construir.

O estabelecimento de processos de Educação Permanente em rede abrangente de atores em atuação sinérgica demanda, por essa perspectiva, estratégias para o envolvimento de gestores, formadores, estudantes, profissionais dos serviços e usuários como autores, sujeitos da construção "na" e "da" rede. Tais alternativas, a respeitar a noção de integração no sentido amplo e includente, certamente serão multirreferenciadas, interdisciplinares e plurais, aumentando ainda mais a complexidade envolvida na construção desse processo de integração.

Ampliar as parcerias exige o fortalecimento da autonomia coletiva e, para tanto, o reconhecimento e a aceitação do outro como alteraçãa $0^{17}$. $O$ termo alteração tem, para o autor, a conotação de movimento e de interferência, em lugar de alteridade - que pode ser relativa somente à "idéia" do outro. No mundo contemporâneo, o enfrentamento da complexidade demanda menos a superação do que a aceitação de sua pluralidade de constituintes heterogêneos, a percepção do "outro" como uma interferência, que impõe aos sujeitos uma experiência de heterogeneidade radical. Nesses termos, a noção de heterogeneidade, mais do que a de diferença, ganha força, reabilitam-se o plural e o heterogêneo e, além, "o caráter finalmente mais normal que patológico do conflito e da alteração, bem como o reconhecimento do tempo e da história para a compreensão dos fenômenos" ${ }^{17}$.

É na perspectiva da heterogeneidade, de pluralidade de olhares irredutíveis entre si, que se abre a proposta de constituição da Rede de Educação Permanente em Atenção Oncológica: na aceitação cuidadosa de um desafio de integração; de abertura de espaços de diálogo de gestores, formadores, profissionais das equipes e população; de difusão de experiências e desenvolvimento de iniciativas para a gestão da educação e do trabalho orientadas para a transformação das práticas em direção à melhoria e à integralidade na atenção oncológica no país.

\section{REFERÊNCIAS}

1. Brasil. Ministério da Saúde. Pacto pela Saúde 2006 Consolidação do SUS Portaria no 399/GM de 22 fevereiro 2006.

2. Brasil. Ministério da Saúde. Diretrizes operacionais dos Pactos pela Vida, em Defesa do SUS e de Gestão. Brasília: Editora MS; 2006.
3. Schwartz Y. The components of competency, a necessary exercise for an unsolvable problem. Education Permanente 1997;(133):9-34.

4. Haddad J, Roschke MA, Davini MC (org). Educación permanente de personal de salud. Washington: OPS/OMS; 1994.

5. Brasil. Ministério da Saúde. Lei no 8.080 de 19 setembro1990.

6. Rovere MR. Planificación estratégica de recursos humanos en salud. Serie Desarrollo de Recursos Humanos n. 96. Washington: Organización Panamericana de la Salud; 1993.

7. Brasil. Ministério da Saúde. Política Nacional de Formação e Desenvolvimento para o SUS: caminhos para a educação permanente em saúde e a estratégia de Pólos ou Rodas de Educação Permanente em Saúde. Resolução MS/CNS nº 335 de 27 novembro 2003.

8. Brasil. Ministério da Saúde. Política Nacional de Atenção Oncológica. Portaria nº 2439/GM de 19 dezembro 2005.

9. Instituto Nacional de Câncer (INCA/MS). Estimativas 2006: Incidência de Câncer no Brasil. Rio de Janeiro: INCA; 2005.

10. Cinterfor-OIT. Formación basada en competencia laboral: situación actual y perspectivas. Montevideo: Conocer; 1997.

11. Freire P. Educação como prática da liberdade. $27^{\mathrm{a}}$ ed. Rio de Janeiro: Paz e Terra; 2003.

12. Régnier KD. Alguns elementos sobre a racionalidade dos modelos Fordista, Taylorista e Toyotista. B Téc Senac [revista eletrônica] 1997;23(2). [acesso em 20 nov 2006]. Disponível em: <http://www.senac.br/INFORMATIVO/ BTS/232/ boltec232d.htm>

13. Régnier KD. Educação, trabalho e emprego numa perspectiva global. B Téc Senac [revista eletrônica] 1997;23(1). [acesso em 20 nov 2006]. Disponível em: <http://www.senac.br/INFORMATIVO/BTS/ 231/boltec 231a.htm>

14. Valle R. Uma nova abordagem para o estudo das competências. In: Valle R (Org). O conhecimento em ação. Rio de Janeiro: Relume Dumará; 2003.

15. Deluiz N. O modelo das competências profissionais no mundo do trabalho e na educação: implicações para o currículo. B Téc Senac [revista eletrônica] 2001;27(3). [acesso em 3 jul 2006]. Disponível em: <http: // www.senac.br/INFORMATIVO/BTS/273/ boltec273b.htm>

16. Freire P. Pedagogia do oprimido. $35^{\mathrm{a}}$ ed. Rio de Janeiro: Paz e Terra; 2003.

17. Ardoino J. A complexidade. In: Morin E (org). A religação dos saberes - o desafio do século XXI. $2^{\text {a }}$ ed. Rio de Janeiro: Bertrand Brasil; 2002. 